\title{
First-Principles Calculations on Phase Transition and Elastic Properties of CoN
}

\author{
He De-Chun*, Peng Yong, Li Su-Yuan and He Yong-Lin \\ College of Physics and Electrical Engineering, Hexi University, Zhangye 734000, China
}

(Received December 7, 2015; in final form May 10, 2016)

\begin{abstract}
The structural phase transition and elastic properties of CoN are investigated by ab initio plane-wave pseudopotential density function theory method. The equilibrium lattice parameters $a_{0}$, elastic constants $C_{i j}$, bulk modulus $B_{0}$ and its derivative $B_{0} \prime$ are calculated. From the usual condition of equal enthalpy, the phase transition of CoN from zinc-blende to rocksalt structure occurs at $35.4 \mathrm{GPa}$ with a volume collapse of about $15.6 \%$, consistent with the calculated result $36 \mathrm{GPa}$ (FP-LDA), but an uncertainty is about $4.4 \mathrm{GPa}$ compared with the $31 \mathrm{GPa}$ (ASA-GGA). All three independent elastic constants, $C_{11}, C_{12}$, and $C_{44}$ for CoN are calculated from direct computation of stresses generated by small strains. Both $C_{12}$ and $C_{44}$ are less sensitive to pressure as compared with $C_{11}$. The calculated conclusions offer theoretical data for the further research of the mechanical properties for CoN.
\end{abstract}

DOI: 10.12693/APhysPolA.130.743

PACS/topics: 62.20.--x, 62.20.de, 62.50.-p, 62.20.dq

\section{Introduction}

Most transition metal (TM) nitrides and carbides have been extensively investigated because they possess a set of unique magnetoelectric and mechanical properties. For example, in the field of high density magnetic recording, iron nitrides are applied [1-5]. These properties make them suitable as bulk or thin film materials in many technological applications. Cobalt-nitride system $\left(\mathrm{Co}_{x} \mathrm{~N}, x=1,2,3,4\right)$, as a high hardness and magnetic storage material, has been extensively reported. A cubic $\mathrm{Co}_{4} \mathrm{~N}$ was prepared with a lattice constant of $6.7788 \mathrm{bohr}$ $(1 \mathrm{bohr}=0.529 \AA)$, and investigated magnetic properties and bonding analyses of perovskite structure $\mathrm{Co}_{4} \mathrm{~N}$ nitride within density functional theory using both pseudopotential and all electron methods [6]. The bulk $\mathrm{Co}_{2} \mathrm{~N}$ was synthesised with an orthorhombic $\mathrm{Fe}_{2} \mathrm{C}$-type structure, but no structure was illustrated in $\mathrm{Co}_{2} \mathrm{~N}$ films $[7,8]$. In the early studies, mononitride $\mathrm{CoN}$ has been proved that it has zinc-blende (ZB) and rocksalt (RS) structures. RS-CoN with a lattice constant of $4.27 \AA$ was prepared by decomposition of $\mathrm{Co}\left(\mathrm{NH}_{2}\right)_{3}$ [9], while the $\mathrm{ZB}-\mathrm{CoN}$ was obtained with a lattice constant of $4.28 \AA$ by decomposing $\left[\mathrm{Co}\left(\mathrm{NH}_{3}\right)_{6}\right]\left(\mathrm{N}_{3}\right)_{3}[10]$. ZB-CoN and ZB-FeN films were grown by reactive sputtering in a mixture of $\mathrm{Ar}+\mathrm{N}_{2}$ gas, and the paramagnetic properties was observed in ZB-CoN $[11,12]$. In addition, cobalt nitride films were deposited by reactive pulsed laser deposition on silicon substrates at room temperature. The difference of 0.5 and $1.5 \mathrm{eV}$ in the binding energy of $\mathrm{Co}_{2 p 3 / 2}$ and $\mathrm{N}_{1 s}$ electrons, respectively, was evidenced, and the reaction between nitrogen and cobalt was effectively made [8]. Mater et al. [6] prepared $\mathrm{Co}-\mathrm{N}$ films by using the rfsputtering method. The sputtered films consist of one

\footnotetext{
* corresponding author; e-mail: dechunhe@126.com
}

or two phase, such as $\mathrm{CoN}, \mathrm{Co}_{2} \mathrm{~N}, \mathrm{Co}_{3} \mathrm{~N}, \mathrm{Co}_{4} \mathrm{~N}$ and $\alpha$-Co. The preferred orientation was observed on the Co-N films. Saturation magnetization $\sigma_{s}$ of Co-N sputtered film decreases from 160 to 17 e.m.u. $\mathrm{g}^{-1}$ with increasing content of $N$ from 0 to 21.7 at.\%. The selfsupported ordered mesoporous cobalt and chromium nitrides were synthesised by Shi et al. [13]. Small-angle $\mathrm{X}$-ray diffraction (XRD) patterns and transmission electron microscopy (TEM) images showed that mesoporous $\mathrm{CoN}$ and $\mathrm{CrN}$ nanowires with a $2 \mathrm{D}$ ordered hexagonal structure were obtained. Wide-angle XRD patterns, high resolution TEM (HRTEM) images and selected area electron diffraction (SAED) patterns revealed the formation of crystallite metal nitrides.

However, to the best of our knowledge, though the CoN has been well known for a long time, its theoretical study is still few, except a few of density function theory calculations on $\mathrm{CoN}$ were carried out [14, 15], for example Liu reports systematic results from ab initio calculations with density functional theory on three cubic structures, zinc blende $(\mathrm{zb})$, rocksalt $(\mathrm{rs})$ and cesium chloride (cc), of the ten $3 d$ transition metal nitrides [16]. Many experimental phenomena could not be explained completely. Hence, the theoretic work on $\mathrm{CoN}$ is quite significative. First principles calculation is one of the most powerful tool for performing theoretical investigation for solid properties [16]. Using first principles density functional theoretical calculations, Soni reports a systematic nonspin and spin polarized total energy calculations of the lattice dynamical and a number of other properties such as band structure, structural and magnetic moment of two mononitrides $\mathrm{FeN}$ and $\mathrm{CoN}$. The phonon dispersion curves and phonon density of states in the case of $\mathrm{FeN}$ and $\mathrm{CoN}$ have been determined [17]. In this work, we focus on the structural phase transition and elastic properties of $\mathrm{CoN}$ by using the first principles calculations with pseudopotential plane-wave method (PP-PW), which is implemented in the computer code fhimd, yields reliable 
predictions for the atomic and electronic structure as well as many physical and chemical properties of a broad range of materials.

\section{Calculated methods}

Pseudopotential plane-wave $a b$ initio calculations were performed within the framework of density functional theory (DFT) [18]. The exchange and correlation function was described by the generalized gradient approximation (GGA) with Perdew-Burke-Ernzerhof (PBE) [19]. The ultrasoft pseudopotential [20] was used to generate the pseudopotentials for $\mathrm{Co}$ and $\mathrm{N}$, respectively. The Co $3 \mathrm{~d}^{7} 4 \mathrm{~s}^{2}$ and $\mathrm{N} 2 \mathrm{~s}^{2} 2 \mathrm{p}^{3}$ electrons were explicitly treated as valence electrons. The integral over the Brillouin zone was $8 \times 8 \times 8 k$-point using the MonkhorstPack special $k$-point approach [21]. The electron wave functions were expanded in plane-wave basis with an energy cutoff of $430 \mathrm{eV}$. To ensure proper convergence of self-consistency calculation, the calculated total energy of the crystal converged to less than $5.0 \times 10^{-6} \mathrm{eV} /$ atom, the maximum residual force less than $0.01 \mathrm{eV} / \AA$, the maximum stress below $0.02 \mathrm{GPa}$ and the displacement of atoms during the geometry optimization less than $5 \times 10^{-4} \AA$. All the total energy calculations were performed using the Cambridge Serial Total Energy Package (CASTEP) code [20, 22].

\section{Results and discussion}

\subsection{The structures and phase transition of $\mathrm{CoN}$}

The ZB and RS are two typical structures for CoN. For the ZB structure, the space group is $F \overline{4} 3 M$ (No. 216), the atomic coordinates are Co $4 \mathrm{a}(0,0,0)$ and N 4c $(0.25,0.25,0.25)$. Similarly, the RS structure has $F M \overline{3} M$ (No. 225) space group, the atomic coordinates are Co $4 \mathrm{a}(0,0,0)$ and $\mathrm{N} 4 \mathrm{~b}(0.5,0.5,0.5)$. For both the ZB and RS structures of $\mathrm{CoN}$, the equilibrium lattice parameters $a_{0}$ are obtained by carrying out geometric optimization, and the total energy $E$ and the corresponding primitive cell volume $V$ are calculated by setting a series of different lattice constants, then the zero pressure bulk modulus $B_{0}$ and its pressure derivative $B_{0} /$ are obtained by fitting the $E-V$ curves with the Birch-Murnaghan equation of state (EOS) [23]. The $E-V$ curves of $\mathrm{CoN}$ are plotted in Fig. 1. It is shown that the $\mathrm{ZB}-\mathrm{CoN}$ is stable compared with RS-CoN at zero pressure, the groundstate energies are -5255.40 and $-5252.71 \mathrm{eV}$ per eight atoms, respectively. Our calculated equilibrium lattice parameters $a_{0}$, elastic constants $C_{i j}$, bulk modulus $B_{0}$ and its derivative $B_{0}$ ' of $\mathrm{CoN}$ at zero pressure and zero temperature are listed in Table I. It is demonstrated that our calculated results are in satisfactory agreement with the experimental data [9-11] and other theoretical results [15].

TABLE I

The lattice constants $[\AA]$, aggregate elastic modulus $[\mathrm{GPa}]$, pressure derivatives of bulk modulus and the elastic constants $[\mathrm{GPa}]$ for ZB- and RS-CoN at $T=0 \mathrm{~K}$ and $P=0 \mathrm{GPa}$.

\begin{tabular}{c|c|c|c|c}
\hline \hline & \multicolumn{2}{|c|}{ ZB } & \multicolumn{2}{c}{ RS } \\
\cline { 2 - 5 } & Present & Other & Present & Other \\
\hline$a_{0}$ & 4.298 & $4.280[10], 4.297[11], 4.182[15], 4.215[15], 4.276[15]$ & 4.042 & $4.270[9], 3.932[15], 3.964[15], 3.972[15]$ \\
$C_{11}$ & 295.4 & - & 431.5 & - \\
$C_{12}$ & 227.7 & - & 237.9 & - \\
$C_{44}$ & 56.7 & - & 84.3 & - \\
$B_{0}$ & 250.3 & $302.0[15], 283.0[15], 294.0[15]$ & 302.4 & $378.0[15], 350.0[15], 352.0[15]$ \\
$\partial C_{11} / \partial P$ & 3.53 & - & 8.60 & - \\
$\partial C_{12} / \partial P$ & 4.30 & - & 2.26 & - \\
$\partial C_{44} / \partial C_{44}$ & -0.52 & - & -0.19 & - \\
$\partial B / \partial P$ & 4.05 & - & 4.37 & - \\
$B_{0}^{\prime}$ & 4.6 & $4.4[15], 4.4[15], 4.9[15]$ & 4.6 & $4.6[15], 4.7[15], 4.6[15]$
\end{tabular}

Table I show the large difference between C11 values of $\mathrm{RS}$ and $\mathrm{ZB}$ CoN because the elastic constant $C_{11}$ represents elasticity in length. Figure 1 shows that the $\mathrm{ZB}$ crystal structure for CoN has the lower energy minimum and that this minimum occurs at a larger lattice constant than for RS. This means that at zero pressure, the ZB is the preferred phase but at higher pressure, a phase transition to the RS phase may be possible. One simple method for obtaining the phase transition pressure of
CoN from the $\mathrm{ZB}$ to $\mathrm{RS}$ structure is the usual condition of equal enthalpies [24]. The phase transition pressure $P_{\mathrm{t}}$ is determined by the Gibbs free energy $G=E+P V+T S$. Since in present work, $T=0 \mathrm{~K}$, the Gibbs free energy becomes equal to the enthalpy $H=E+P V$. The calculated enthalpy $H$ as a function of pressure $P$ is illustrated in Fig. 2a. It is indicated that the phase transition from the $\mathrm{ZB}$ to the RS phase of $\mathrm{CoN}$ is about $35.4 \mathrm{GPa}$. Slightly different values are obtained for this transition 


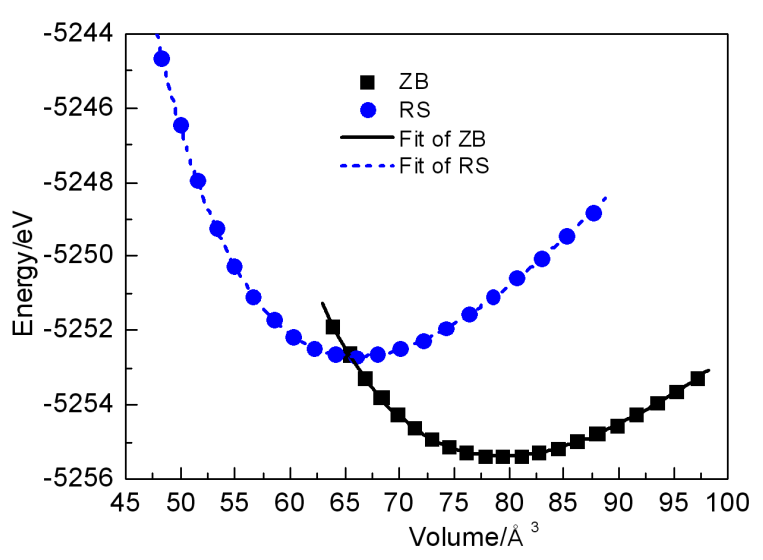

Fig. 1. Total energy as a function of volume for ZBand RS-CoN.

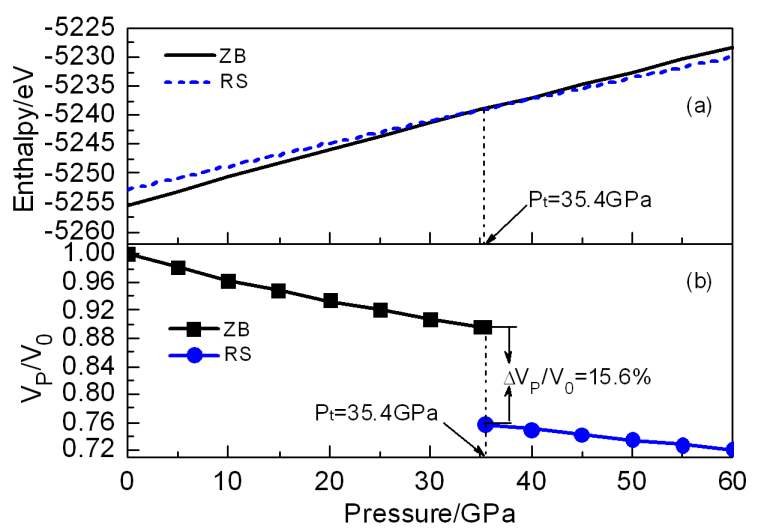

Fig. 2. Enthalpy (a) and normalized volume (b) as a function of pressure for the $\mathrm{ZB}$ and $\mathrm{RS}$ structures of $\mathrm{CoN}$.

pressure when using the FT-LDA method (36 GPa) [15], and the uncertainty is about $4.4 \mathrm{GPa}$ compared with the ASA-GGA method (31 GPa) [15]. The lattice dynamic instabilities have been considered to be responsible for the phase transition with drastic atomic motion and lattice distortion induced by the pressure. The normalized volume $V_{\mathrm{P}} / V_{0}$ versus pressure for the $\mathrm{ZB}$ and $\mathrm{RS}$ phase of $\mathrm{CoN}$ is plotted in Fig. 2b. The normalized volume $V_{\mathrm{P}} / V_{0}$ for the $\mathrm{ZB}$ structure is $V_{\mathrm{ZB}}\left(P_{\mathrm{t}}\right) / V_{0}=0.896$ and that for $\mathrm{RS}$ is $V_{\mathrm{RS}}\left(P_{\mathrm{t}}\right) / V_{0}=0.757$ when the phase transition occurs, where $V_{0}$ is the ground-state volume of ZB-CoN, and the volume collapse $\Delta V_{\mathrm{P}} / V_{0}$ is about $15.6 \%$.

\subsection{Elastic properties}

Elastic properties are very important for materials because they provide information on interatomic potentials and relate to various fundamental solid state phenomena, such as interatomic bonding, equation of state, phonon spectra as well as specific heat, thermal expansion, the Debye temperature and the Grüneisen parameter $[25,26]$. The elastic constants are obtained by means of a Taylor expansion of the total energy. The derivative of energy as a function of a lattice strain is as follows [27]:

$$
\begin{aligned}
& E(V, \delta)=E\left(V_{0}, 0\right)+V_{0}\left(\sum_{i} \tau_{i} \delta_{i} \xi_{i}\right. \\
& \left.+\frac{1}{2} \sum_{i j} C_{i j} \delta_{i} \xi_{i} \delta_{j} \xi_{j}\right),
\end{aligned}
$$

where $E(V, 0)$ is the energy of the unstrained system with equilibrium volume $V_{0}, \tau_{i}$ is an element of stress tensor and $\xi_{i}$ is a factor of the Voigt index. In present work, for all strain, $\delta= \pm 0.0018, \pm 0.003, \pm 0.0006$ are taken to calculate the total energy $E$ for $\mathrm{ZB}$ - and RS-CoN, respectively.

Since the strain and stress tensors are symmetric, the general elastic stiffness tensor has only 21 non-zero independent components. For a cubic crystal, they are reduced to three elastic moduli, $C_{11}, C_{12}$ and $C_{44}$ fully describe its elastic behavior. $C_{11}$ and $C_{12}$ can be determined from the bulk modulus $B$ and the shear constant $c$ :

$$
\begin{aligned}
& B=\left(C_{11}+2 C_{12}\right) / 3, \\
& c=\left(C_{11}-C_{12}\right) / 2,
\end{aligned}
$$

where the shear constant $c$ describes materials resistance to shear deformation across the (110) plane in the [110] direction, and $C_{44}$ is the resistance to shear deformation across the (100) plane in the [010] direction. Besides the bulk modulus $B$, the shear modulus $G$ (in the Voigt notation) can be defined by the following equation [28-30]:

$$
G=\left(G_{\mathrm{V}}+G_{\mathrm{R}}\right) / 2,
$$

where $G_{\mathrm{V}}=\left(C_{11}-C_{12}+3 C_{44}\right) / 5$ and $G_{\mathrm{R}}=\left[5\left(C_{11}-\right.\right.$ $\left.\left.C_{12}\right)\right] /\left[4 C_{44}+3\left(C_{11}-C_{12}\right)\right]$ are the Voigt shear modulus and the Reuss shear modulus, respectively.

To study the stability of ZB- and RS-CoN, we calculated the elastic constants $C_{i j}$, bulk modulus $B$, the shear modulus $G$, the Voigt shear modulus $G_{\mathrm{V}}$ and the Reuss shear modulus $G_{\mathrm{R}}$ at zero pressure and temperature, and the results are listed in Tables II and III. Although there are no experimental or theoretical data available to compare the elastic constants, our data will be beneficial to the future investigation. For cubic crystals, the mechanical stability criteria are given by [31]:

$$
\tilde{C}_{\alpha \alpha}>0, \quad \tilde{C}_{11}>\left|\tilde{C}_{12}\right|, \quad \tilde{C}_{11}+2 \tilde{C}_{12}>0,
$$

where $\tilde{C}_{\alpha \alpha}=C_{\alpha \alpha}-P(\alpha=1,4), \tilde{C}_{12}=C_{12}+P$. So we can confirm that the mechanical properties of ZB- and RS-CoN are stable at zero pressure and temperature. We can estimate that the phase transition from the $\mathrm{ZB}$ phase to RS phase for $\mathrm{CoN}$ occurs at $40.0 \mathrm{GPa}\left(C_{44}-P=37.3-40=-2.7 \mathrm{GPa}<0\right)$. This result gets close to $35.4 \mathrm{GPa}$ and Lukashev results $(31 \mathrm{GPa}$ and $36.0 \mathrm{GPa}$ ). In order to analyze the tendency of elastic properties for ZB- and RS-CoN with the pressure increasing in detail, the range of pressures from 0 to $60 \mathrm{GPa}$ is considered.

The elastic moduli as a function of pressure for ZB- and RS-CoN are shown in Fig. 3a and b, respectively. It is found that the elastic constants $C_{11}, C_{12}$ and bulk modulus $B$ increase when the pressure is enhanced and a linear dependence in all curves of $\mathrm{CoN}$ in the considered range of pressure, but the variations of $C_{44}$ with the pressure 


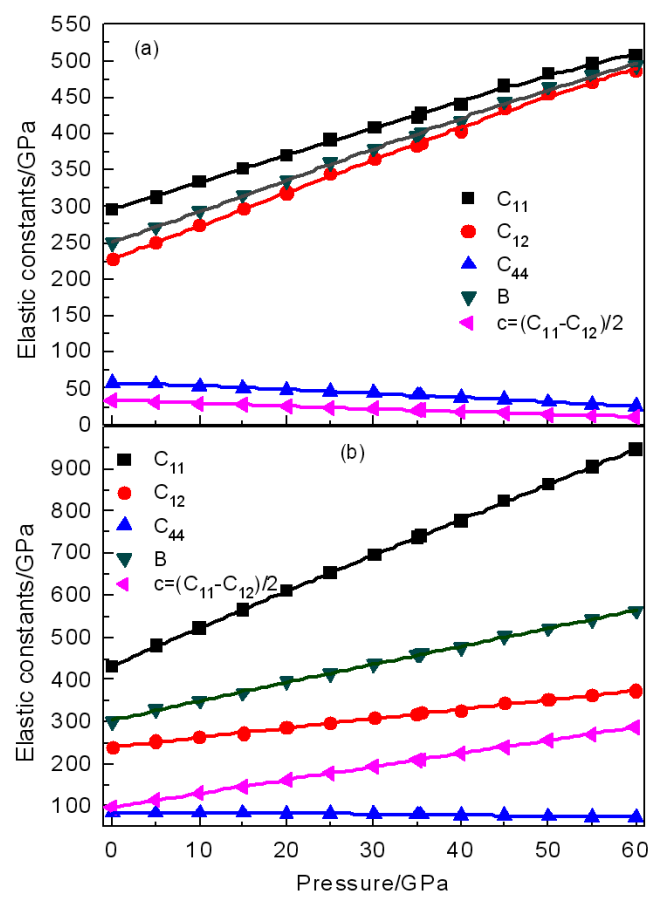

Fig. 3. Elastic constants $C_{i j}$, bulk modulus $B$ and shear constant $c$ as functions of pressure: (a) ZB-CoN and (b) RS-CoN, the solid lines are fitting curves.

TABLE II

Elastic constants $C_{i j}$, bulk modulus $B$, shear modulus $G$, Voigt shear modulus $G_{\mathrm{V}}$, Reuss shear $G_{\mathrm{R}}$, density $\rho$, isotropic wave velocities and Debye temperature $\Theta_{\mathrm{D}}$ dependence on pressure $P$ for $\mathrm{ZB}-\mathrm{CoN}$.

\begin{tabular}{c|c|c|c|c|c|c|c|c|c|c|c|c}
\hline \hline$P$ & $\rho$ & $C_{11}$ & $C_{12}$ & $C_{44}$ & $B$ & $G_{\mathrm{V}}$ & $G_{\mathrm{R}}$ & $G$ & $v_{p}$ & $v_{s}$ & $v_{m}$ & $\Theta_{\mathrm{D}}$ \\
\hline 0 & 6.10 & 295.4 & 227.7 & 56.7 & 250.3 & 47.5 & 44.6 & 46.1 & 7.15 & 2.75 & 3.12 & 431.8 \\
5 & 6.22 & 313.0 & 249.5 & 55.5 & 270.7 & 46.0 & 42.7 & 44.4 & 7.28 & 2.67 & 3.03 & 422.9 \\
10 & 6.33 & 333.9 & 274.3 & 52.6 & 294.2 & 43.5 & 40.3 & 41.9 & 7.43 & 2.57 & 2.92 & 410.1 \\
15 & 6.43 & 352.8 & 297.1 & 50.5 & 315.6 & 41.5 & 38.1 & 39.8 & 7.57 & 2.49 & 2.83 & 399.0 \\
25 & 6.63 & 391.9 & 344.1 & 46.1 & 360.0 & 37.2 & 33.6 & 35.4 & 7.84 & 2.31 & 2.63 & 375.2 \\
30 & 6.72 & 408.0 & 364.2 & 43.4 & 378.8 & 34.8 & 31.2 & 33.0 & 7.93 & 2.22 & 2.53 & 361.5 \\
35 & 6.80 & 422.8 & 382.6 & 40.0 & 396.0 & 32.0 & 28.7 & 30.4 & 8.01 & 2.11 & 2.41 & 346.2 \\
35.4 & 6.81 & 427.8 & 388.0 & 39.8 & 401.3 & 31.8 & 28.4 & 30.1 & 8.05 & 2.10 & 2.40 & 344.9 \\
40 & 6.89 & 440.4 & 404.0 & 37.3 & 416.1 & 29.6 & 26.2 & 27.9 & 8.11 & 2.01 & 2.30 & 331.7 \\
45 & 6.97 & 465.9 & 433.5 & 34.4 & 444.3 & 27.1 & 23.7 & 25.4 & 8.28 & 1.91 & 2.18 & 316.0 \\
50 & 7.04 & 483.7 & 455.1 & 31.3 & 464.6 & 24.5 & 21.3 & 22.9 & 8.38 & 1.80 & 2.06 & 299.2 \\
55 & 7.12 & 496.3 & 471.2 & 28.5 & 479.5 & 22.1 & 18.9 & 20.5 & 8.44 & 1.70 & 1.94 & 283.0 \\
60 & 7.20 & 507.5 & 486.0 & 25.4 & 493.2 & 19.5 & 16.4 & 18.0 & 8.48 & 1.58 & 1.81 & 264.5
\end{tabular}

is very slight. The elastic constant $C_{11}$ represents elasticity in length. A longitudinal strain produces a change in $C_{11}$. The elastic constants $C_{12}$ and $C_{44}$ are related to the elasticity in shape, which are two shear constants. A transverse strain causes a change in shape but without a change in volume. Hence, $C_{12}$ and $C_{44}$ are less sensitive to pressure as compared with $C_{11}$. The pressure derivatives $\partial C_{11} / \partial P, \partial C_{12} / \partial P, \partial C_{44} / \partial P$ and $\partial B / \partial P$, for ZBand RS-CoN are also calculated, and the results are listed in Table I. In order to estimate the distortion in a certain plane of cubic crystal, the shear moduli $c$ and $c l=C_{44}$, corresponding to shear along the (11) and (100) planes, respectively, are calculated. As shown in Fig. 3a and b, the changes of $c$ and $c$ for ZB-CoN are slight with pressure, which shows that pressure will not cause obvious variation of shear along the (11) and (10) planes. The change of $c$ for RS-CoN is also slight with the increase of pressure, but the change of $c$ increases obviously. It indicates that pressure will not cause obvious variation of shear along the (110) plane, but the pressure will make changes for shear along the (100) plane.

TABLE III

Elastic constants $C_{i j}$, bulk modulus $B$, shear modulus $G$, Voigt shear modulus $G_{\mathrm{V}}$, Reuss shear $G_{\mathrm{R}}$, density $\rho$, isotropic wave velocities and temperature $\Theta_{\mathrm{D}}$ dependence on pressure $P$ for RS-CoN.

\begin{tabular}{c|c|c|c|c|c|c|c|c|c|c|c|c}
\hline \hline$P$ & $\rho$ & $C_{11}$ & $C_{12}$ & $C_{44}$ & $B$ & $G_{\mathrm{V}}$ & $G_{\mathrm{R}}$ & $G$ & $v_{p}$ & $v_{s}$ & $v_{m}$ & $\Theta_{\mathrm{D}}$ \\
\hline 0 & 7.33 & 431.5 & 237.9 & 84.3 & 302.4 & 89.3 & 88.9 & 89.1 & 7.58 & 3.48 & 3.93 & 578.4 \\
5 & 7.46 & 480.3 & 252.7 & 84.0 & 328.5 & 95.9 & 93.9 & 94.9 & 7.81 & 3.57 & 4.02 & 595.5 \\
10 & 7.57 & 523.1 & 262.7 & 83.4 & 349.5 & 102.1 & 97.4 & 99.8 & 7.98 & 3.63 & 4.09 & 609.2 \\
15 & 7.68 & 565.4 & 272.7 & 82.8 & 370.3 & 108.2 & 100.2 & 104.2 & 8.14 & 3.68 & 4.15 & 621.2 \\
20 & 7.78 & 609.9 & 285.5 & 82.0 & 393.6 & 114.0 & 102.2 & 108.1 & 8.31 & 3.73 & 4.21 & 631.7 \\
25 & 7.88 & 652.6 & 296.3 & 81.2 & 415.1 & 120.0 & 103.8 & 111.9 & 8.46 & 3.77 & 4.25 & 641.5 \\
30 & 7.97 & 696.4 & 308.7 & 80.3 & 437.9 & 125.7 & 104.9 & 115.3 & 8.62 & 3.80 & 4.29 & 650.3 \\
35 & 8.06 & 737.5 & 318.4 & 79.3 & 458.1 & 131.4 & 105.5 & 118.5 & 8.74 & 3.83 & 4.33 & 658.0 \\
35.4 & 8.07 & 741.1 & 319.6 & 79.1 & 460.1 & 131.8 & 105.5 & 118.6 & 8.76 & 3.84 & 4.33 & 658.4 \\
40 & 8.14 & 775.6 & 326.3 & 77.9 & 476.1 & 136.6 & 105.4 & 121.0 & 8.84 & 3.85 & 4.35 & 664.0 \\
45 & 8.23 & 823.5 & 342.8 & 76.6 & 503.0 & 142.1 & 105.3 & 123.7 & 9.01 & 3.88 & 4.38 & 670.6 \\
50 & 8.31 & 863.2 & 351.5 & 75.4 & 522.1 & 147.6 & 105.0 & 126.3 & 9.12 & 3.90 & 4.41 & 676.6 \\
55 & 8.39 & 904.6 & 362.2 & 74.0 & 543.0 & 152.9 & 104.4 & 128.6 & 9.23 & 3.92 & 4.43 & 682.0 \\
60 & 8.46 & 947.2 & 373.4 & 72.9 & 564.7 & 158.5 & 103.9 & 131.2 & 9.35 & 3.94 & 4.45 & 687.9
\end{tabular}

\section{Conclusion}

We have reported the results of phase transition and elastic properties for ZB- and RS-CoN using the $a b i n i$ tio plane-wave pseudopotential density function theory method, on which few papers are presented. From the usual condition of equal enthalpy, the phase transition of $\mathrm{CoN}$ from zinc-blende to rocksalt structure occurs at $35.4 \mathrm{GPa}$ with a volume collapse of about $15.6 \%$, consistent with the calculated result $36 \mathrm{GPa}$ (FP-LDA), but an uncertainty is about $4.4 \mathrm{GPa}$ compared with the $31 \mathrm{GPa}$ (ASA-GGA). The tendencies of elastic properties for $\mathrm{CoN}$ are analyzed over a pressure range from 0 to $60 \mathrm{GPa}$. The calculated conclusions offer theoretical data for the further research of the mechanical properties for $\mathrm{CoN}$.

\section{References}

[1] D. Andriamandroso, G. Demazeau, M. Pouchard, P. Hagenmuller, J. Solid State Chem. 54, 54 (1984).

[2] S. Matar, P. Mohn, G. Demazeau, B. Siberchicot, J. Phys. (France) 49, 1761 (1988).

[3] S. Ishida, K. Kitawatase, J. Magn. Magn. Mater. 104, 1933 (1992).

[4] A. Sakuma, J. Magn. Magn. Mater. 88, 369 (1990).

[5] R. Coehoorn, G.H.O. Daalderop, H.J.F. Jansen, Phys. Rev. B 48, 3830 (1993).

[6] S.F. Matar, A. Houari, M.A. Belkhir, Phys. Rev. B 75, 245109 (2007). 
[7] M. Hasegawa, T. Yagi, Solid State Commun. 135, 294 (2005).

[8] W. De La Cruz, O. Contreras, G. Soto, E. PerezTijerina, Rev. Mex. Fis. 52, 409 (2006).

[9] O. Schmidt-Dumont, N. Kron, Angew. Chem. 67, 231 (1955).

[10] T.B. Joyner, F.H. Verhoek, J. Am. Chem. Soc. 83, 1069 (1961)

[11] K. Suzuki, T. Kaneko, H. Yoshida, H Morita, H. Fujimori, J. Alloys Comp. 224, 232 (1995).

[12] K. Suzuki, H. Morita, T. Kaneko, H. Yoshida, H. Fujimori, J. Alloys Comp. 201, 11 (1993).

[13] Yifeng Shi, Ying Wan, Renyuan Zhang, Dongyuan Zhao, Adv. Funct. Mater. 18, 2436 (2008).

[14] H. Shimizu, M. Shirai, N. Zuzuki, J. Phys. Soc. Jpn. 67, 922 (1998).

[15] P. Lukashev, W.R.L. Lambrecht, Phys. Rev. B 70, 245205 (2004).

[16] Z.T.Y. Liu, X. Zhou, S.V. Khare, D. Gall, J. Phys. Condens. Matter 26, 025404 (2014).

[17] H.R. Soni, V. Mankad, S.K. Gupta, P.K. Jha, J. Alloys Comp. 522, 106 (2012).

[18] W. Kohn, L.J. Sham, Phys. Rev. 140, A1133 (1965).

[19] J.P. Perdew, K. Burke, M. Ernzerhof, Phys. Rev. Lett. 77, 3865 (1996).
[20] V. Milman, B. Winkler, J.A. White, C.J. Packard, M.C. Payne, E.V. Akhmatskaya, R.H. Nobes, Int. J. Quantum Chem. 77, 895 (2000).

[21] J.D. Pack, H.J. Monkhorst, Phys. Rev. B 16, 1748 (1977).

[22] M.C. Payne, M.P. Teter, D.C. Allan, T.A. Arias, J.D. Joannopoulos, Rev. Mod. Phys. 64, 1045 (1992).

[23] F. Birch, Phys. Rev. 71, 809 (1947).

[24] L. Liu, J.J. Wei, X.Y. An, X.M. Wang, H.N. Liu, W.D. Wu, Chin. Phys. B 20, 106201 (2011).

[25] C.A. Ponce, R.A. Casali, M.A. Caravaca, J. Phys. Condens. Matter 20, 045213 (2008).

[26] A. Bouhemadou, R. Khenata, M. Chegaar, S. Maabed, Phys. Lett. A 371, 337 (2007).

[27] L. Fast, J.M. Wills, B. Johansson, O. Eriksson, Phys. Rev. B 51, 17431 (1995).

[28] W. Voigt, Lehrbuch der Kristallphysik (Handbook of Crystal Physics), Teubner, Leipzig 1928.

[29] A. Reuss, Z. Angew. Math. Mech. 9, 49 (1929).

[30] R. Hill, Phys. Soc. London 65, 350 (1952).

[31] G.V. Sin'ko, N.A. Smirnov, J. Phys. Condens. Matter 14, 6989 (2002). 